\title{
A BIDIRECTIONAL MODEL FOR NATURAL LANGUAGE PROCESSING
}

\author{
Günter Neumann \\ Lehrstuhl für Computerlinguistik \\ Universität des Saarlandes \\ Im Stadtwald 15, Bau 17.2 \\ 6600 Saarbrücken 11, FRG \\ neumann@coli.uni-sb.de
}

\begin{abstract}
In this paper* I will argue for a model of grammatical processing that is based on uniform processing and knowledge sources. The main feature of this model is to view parsing and generation as two strongly interleaved tasks performed by a single parametrized deduction process. It will be shown that this view supports flexible and efficient natural language processing.
\end{abstract}

\section{INTRODUCTION}

The aspect of bidirectionality has been gaining importance since the growing rate of research on natural language generation over the last years offers us deeper insights into this cognitive ability of humans. There are theoretical as well as practical reasons for adopting bidirectionality. Theoretically, the assumption of common knowledge sources for both generation and analysis is essential for the view of language as "an interpersonal medium and an interface to thought" (McDonald 1987). From a psychological point of view, there is a certain amount of empirical evidence for shared processors or facilities (Jackendoff 1987). From a system engineering view, a bidirectional system produces utterances only from that subset of language that it is capable to understand. Therefore, inconsistencies of the language behaviour of the system can be avoided (Jacobs 1988).

A fundamental requirement of a bidirectional knowledge base is that it be represented declaratively (Appelt 1987). From this viewpoint one can distinguish two different types of bidirectional natural language systems:

\footnotetext{
* Thanks to Klaus Netter, Karel Oliva, Norbert Reithinger, Harald Trost and Hans Uszkoreit for fruitful discussions about the aspects of the paper's contents.
}

- systems that use uniform knowledge sources, but different processes

- systems that use uniform knowledge sources as well as uniform processes ${ }^{1}$

Up to now, systems that are capable of analysing and producing language fall into the first class, i.e. they use different operations for both directions (cf. Hoeppner et al. 1983; Busemann and Hauenschild 1988; Allgayer et al. 1989) Currently, it is an open question what degree of bidirectionality should or could be desired (cf. Appelt 1987; Mann 1987; McDonald 1987; Shieber 1988; Jacobs 1988). One of the reasons could be that the formal specification of some tasks (e.g., the determination of content in generation) is currently not well understood in order to decide whether they could be bidirectional in principle.

But in some research areas uniform processing models have been developed that are based on formalisms which are well suited for uniform representation and processing, e.g., Koskenniemi's (1984) two-level model of morphology. Recently, there are first approaches to uniform architectures for grammatical processing (e.g., Shieber 1988; Dymetman and Isabelle 1988; Dymetman et al. 1990). These architectures are based on Pereira and Warren's (1983) paradigm of parsing as deduction. In principle, parsing and generation are viewed as a single parametrized deduction process.

\section{PROBLEMS OF BIDIRECTIONAL GRAMMATICAL PROCESSING}

\footnotetext{
${ }^{1}$ Besides these two classes there are also systems that use different knowledge sources that are compiled from the same source (e.g., Horacek and Pyka 1988) and systems that use common basic representation devices (e.g., Lancel et al. 1988; Neumann and Finkler 1990).
} 
Currently developed approaches that consider parsing as well as generation (e.g., Shieber 1988; Shieber et al. 1990; Dymetman et al. 1990; van Noord 1990; Zajac and Emele 1990) assume:

- that both tasks take place independently from each other, i.e. an utterance is either generated or parsed and

- that grammatical processing can be performed without considerations of discourse.

A great problem with this view is that it offers no solution of the problem of choice between paraphrases in generation: The proposed approaches assume - more or less explicitly modularity between the conceptual and grammatical component of a natural language system. ${ }^{2}$ A great advantage of a modular design especially for uniform architectures is that it is possible to view the grammatical component as relatively autonomous and selfcontained (cf. Appelt 87).

But then the following problems emerge: The conceptual component will be unable to exactly specify the logical form as input to the grammatical component that will precisely lead to the utterance that reflects the intended meaning unless the conceptual module has detailed information about the grammar and knows when to use a specific construction (which renders the modular design meaningless).

On the other hand, when parsing and generation are performed within the grammatical component by a single process only then the opposite view of computing all possible parses of an utterance is the computation of all possible paraphrases of a logical form. When grammatical processing should be modelled by means of a bidirectional grammar, the declarative structure of the grammar must not contain pragmatical or stylistical information because of the modular design. But then the process can only choose randomly between paraphrases during generation and this means that the intended meaning will possibly not be conveyed.

Ideally, a logical language would be helpful which necessarily and sufficiently represents all meaning distinctions of natural

\footnotetext{
2By a conceptual component I mean either the whatto-say component of a generation system or the component that performs inference, plan recognition or anaphora resolution of an understanding system.
}

language. But as Shieber (1988) states "this ... is just the central problem of knowledge representation for natural language in general". Currently, there exist only approximate solutions to this problem for example the use of canonical logical forms (cf. Shieber 88). ${ }^{3}$ But this still offers no solution of the problem of choice between paraphrases.

In this paper it will be argued that the following two points will contribute to an approximate solution:

- interleaved parsing and generation

- using the language use of interlocutors as an additional access criterion to linguistic knowledge

Interleaved parsing and generation means that both tasks take place in parallel (see section 2 ). In principle this results in a bidirectional and incremental flow of information during natural language processing (see section 4.1). An important point during the use of language is that the choice of linguistic material is influenced by the language use of others (see section 3). This leads to more flexibility: not all necessary parameters (e.g., pragmatical values) need to be specified in the input of a generator because decision points can also be set dynamically during run-time.

A promising approach to realize these two features will be to base grammatical processing on a uniform process that is parametrized by means of a declaratively specified preference structure of knowledge sources. But, it is necessary to be aware that the grammatical component must be assumed to be an integrated part of a whole natural language system (in particular in models for performing dialogs) in order to realize this solution.

Before the architecture of the model will be described in section 4 the two issues are explained in more detail in the next sections.

\section{INTERLEAVING GENERATION AND ANALYSIS}

The strategy of viewing natural language processing as based on a uniform deduction process has a formal elegance and results in more compact systems. There is one further advantage that is of both theoretical and practical relevance: uniform architectures offer the possibility to view generation and parsing as strongly interleaved tasks. By this I mean

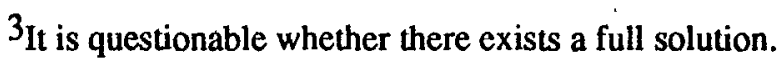


that during performing one task (e.g., generation) the other one (e.g., analysis) is used for monitoring the former. In principle this results in a bidirectional and incremental flow of information:

- During the parse of an utterance the addressee of the utterance can simultaneously start to construct his answer. In doing so, partial results of the parsing process can be used directly during generation (e.g., if a paraphrase will be generated). In such flow of control it will be possible that generation can be used for completing the resulting structure of elliptic, underspecified or ill-formed input during the process of understanding or for generating paraphrases in due time.

- During generation interleaved parsing could help to avoid the construction of ambiguous utterances. E.g., it is necessary for a natural language help system to generate utterances that reflect exactly the intended meaning (if possible at all) to be sure that the dialog partner will perform the correct operations. For instance, producing the utterance "Remove the folder by means of the system tools" is better than "Remove the folder with the system tools" because for the latter utterance there exists the reading "Remove the folder that contains the system tools", too.

Of course, it is also possible to analyse a generated utterance if processes are performing their tasks in an isolated way. ${ }^{4}$ In such flow of control the complete structure has to be generated again if ambiguities are detected that have to be avoided. Because the source of an ambiguous utterance is not used directly to guide the generation process it is possible that the newly generated structure is still ambiguous (and it may happen that the same ambiguous structure is generated again). This results in inefficient systems because in general the loop between the isolated processes must be performed several times.

The advantage of a uniform architecture is that intermediate results of one direction can

\footnotetext{
${ }^{4}$ For example, the complete structure of a produced utterance is analysed during the 'anticipation-feedbackloop' of the HAM-ANS system (see Hoeppner et al. 1983) to detcrmine whether it can be actually uttered clliptic or not.
}

immediately be used in the opposite direction to determine the ambiguous information in due time.

\section{BIDIRECTIONALITY SUPPORTS FLEXIBLE AND EFFICIENT GENERATION}

One of the disadvantages of currently developed generation systems is that they view the structure of linguistic knowledge only statically. If alternatives exist for a particular linguistic expression, decision points are evaluated to determine the appropriate actual utterance. It is necessary to specify corresponding decision points for all possible utterances otherwise the choice must be performed randomly (the determination of the appropriate set of decision points is one of the sources of complexity in existing generation systems). The flexibility of such systems depends directly on the flexibility that is brought into the system via the decision points that are specified by hand during the development of a generation system (i.e. the flexibility is restricted).

On the other side, in a bidirectional system the resulting structures of the parsing task can be used directly during generation. E.g., in general a set of alternative lexemes is specified during the process of lexical choice which are synonymous in the actual situation or when the semantic input cannot be sufficiently specified (e.g., in German, some drinkingdevices can be denoted either 'Tasse' (cup) or 'Becher' (múp) because their shape cannot be interpreted unequivocally). An appropriate choice would be to use the same lexeme that was previously used by the hearer (if no other information is available). In principle this is also possible for the choice between alternative syntactic structures.

This means that uniform architectures offer the possibility to model the assumption that during communication the use of language of one interlocutor is influenced by means of the language use of the others. This adaptability to the use of language of partners in communication is one of the sources for the fact that the global generation process of humans is flexible and efficient. Of course, adaptability is also a kind of co-operative behaviour. This is necessary if new ideas have to be expressed for which no mutually known linguistic terms exist (e.g., during communication between experts and novices). In this case adaptability to the use of language 
of the hearer is necessary in order to make possible that the hearer will be able to understand the new information.

I do not want to argue that all choices are determined by means of language use of others. But, when structures that are determined during analysis are considered during generation, the number of decision points or parameters which have to be specified during the development of a generation system is reduced. This leads to more flexibility: not all necessary parameters need to be specified in the input of a generator because decision points can also be set dynamically during run-time.

This dynamic behaviour of a generation system will increase efficiency, too. As McDonald et al. (1987) define, one generator design is more efficient than another, if it is able to solve the same problem with fewer steps. They argue that "the key element governing the difficulty of utterance production is the degree of familiarity with the situation". The efficiency of the generation process depends on the competence and experience one has acquired for a particular situation. In such situations the generation process performs its task by using compiled knowledge and preferences.

Currently, it is a great problem how compiled knowledge is acquired dynamically and how it is activated in particular situations. But a uniform architecture as proposed in this paper seems to be a promising basis for designing such a system, because the structures determined during analysis could be used for restricting the potential search space.

\section{AN OUTLINE OF A BIDIRECTIONAL ARCHITECTURE}

If both aspects - interleaving parsing and generation and using the language use of interlocutors as additional criterion for the structure of linguistic knowledge - are realized within a uniform architecture then this will increase flexibility and efficiency in natural language processing. E.g., when starting the generation from a logical form, the grammatical process is able to call the conceptual module's attention if a subphrase causes ambiguity. Thus it is not necessary that the conceptual module has detailed information about the grammar.

The flow of control within a system based on an interleaved approach is bidirectional. E.g., during the generation of an utterance partial structures are analysed to avoid unnecessary ambiguities. The bidirectional flow of control supports incremental processing: it is possible to start processing of partial structures before the whole structure is known. In Finkler and Neumann (1989) and Neumann and Finkler (1990) we have already described an implemented generation system (named POPEL-HOW) that realizes an incremental and bidirectional flow of control based on a uniform parallel processing model. The incremental and bidirectional flow of control has two main advantages during generation. Firstly, the determination of contents can be done on the basis of conceptual considerations only, because POPEL-HOW is flexible enough to handle underspecified input. Secondly, the conceptualizer has to regard feedback from POPEL-HOW during the computation of the further selection process. This means, an incremental system like POPEL can model the influence of linguistic restrictions on the process that determines what to say next.

Underspecified structures are analysed in POPEL-HOW at each level of description by means of declarative described mapping rules. The analysis of such structures is performed with generation specific operations. If the system would be based on a uniform architecture then such specific operations are no more necessary.

\subsection{BiLD - A MODEL FOR BIDRECTIONAL LINGUISTIC DEDUCTION}

At the University of Saarbriicken a project called BiLD is now being started where it will be investigated how interleaving of parsing and generation can be efficiently performed and how such a model can be used for increasing flexibility and efficiency during natural language processing. Fig. 1 (next page) shows the schematic structure of its architecture.

The core of the system is a uniform parametrized deduction process. The main task for the process in both directions is the determination of the corresponding syntactic information that functions as an interface between graphematic and semantic information (a formalism based on Headdriven Phrase Structure Grammar (Pollard and Sag 1987) will be used). 


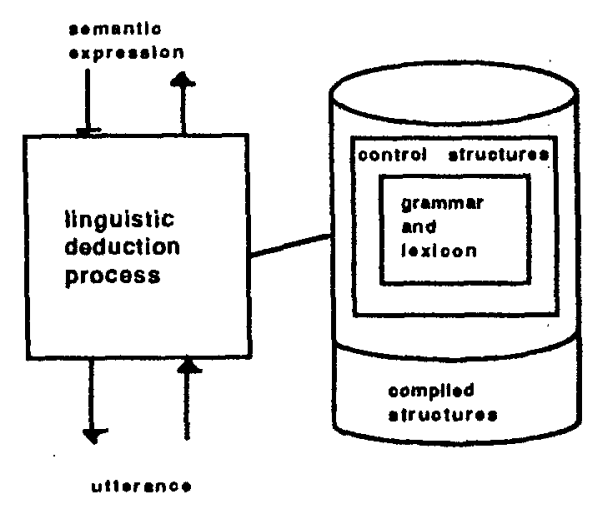

Fig. 1: Schematic structure of BiLD

The task of the deduction process during generation is to construct the graphematic form of a specified semantical feature description. 5 For example, to yield the utterance "A man sings" the deduction process gets as input the semantic feature structure

$$
\left[\operatorname{sem}:\left[\begin{array}{l}
\text { rel : sing' } \\
\text { agens : }\left[\begin{array}{l}
\text { quant : exist' } \\
\text { var : } 7 \\
\text { restr : }\left[\begin{array}{l}
\text { pred }: \text { man' } \\
\text { var }: 7
\end{array}\right.
\end{array}\right]
\end{array}\right]\right]
$$

and deduces the graphematic structure

$$
\text { [graph : }\langle\text { A_man_sings. }\rangle]
$$

by means of successive application of lexical and grammatical information. In the same way the deduction process computes from the graphematic structure an appropriate semantic structure in parsing direction.

The author has now started to develop and implement a first version of a prototype of a uniform algorithm for HPSG. The main idea ${ }^{6}$ is that the approach is head-driven in both directions. In the first phase of the algorithm the maximal projection for all head elements are computed (or predicted) bottom-up. Phrases are then combined top-down. The completion step is controlled by syntactic and semantic information inherited from lexical heads and by the principles of HPSG.

\footnotetext{
${ }^{5}$ The resulting structure of the generation process as well as the input structure of the parsing process is written language, therefore we use the feature 'graph' instead of 'phon' which is preferably used in Pollard and Sag (1987).

${ }^{6}$ Basic ideas of the approach are influenced by the head-driven parser of Proudian and Pollard (1985).
}

Because heads are processed first the completion of structures must be performed in left as well as in right direction.

The approach supports the ID/LP format of rules. But it is an open question whether linear precendence can be processed in the same way for generation and parsing. The problem is that during parsing the task of LP rules is to filter out ungrammatical structures. During generation the task of LP rules can be seen as an ordering criterion. But in this case the problem of choice between paraphrases emerges. In POPEL-HOW it is assumed that the order of activation of concepts (which is determined using pragmatical knowledge) should be maintained if it is syntactically wellformed; otherwise the segments are reordered. Whether such viewpoint is acceptable for generation in general is still open.

\subsection{ASPECTS OF CONTROL STRUCTURE}

A major aspect of the BiLD project is that specific parametrization of the deduction process is represented in the lexicon as well as in the grammar to obtain efficient structures of control (Uszkoreit 1991). The main idea is that preference values are assigned to the elements (disjuncts or conjuncts) of feature descriptions. For example, in HPSG all lexical entries are put together into one large disjunctive form. From a purely declarative point of view these elements are unordered. But a preference structure is used during processing in order to guide the process of lexical choice efficiently which itself influences the grammatical process.

To support flexibility and efficiency (in the way described in section 3) the language use of interlocutors will be considered to influence the preference values. For example, the frequency of access of a lexeme will increase its preference value. In a uniform lexicon it is no matter whether the lexeme was accessed during parsing or generation. But this means that the use of particular linguistic elements of the interlocutor influences the choice of lexical material during generation.

\section{5}

\section{CONCLUSION}

In this paper it is argued that generation and parsing should be best viewed as two interleaved tasks based on à single parametrized deduction process and that this view supports flexible and efficient natural 
language processing. A major point of view is that the language use of interlocutors should be considered during generation as an additional access criterion.

\section{REFERENCES}

Allgayer J.; Jansen-Winkeln R.; Reddig C. and Reithinger N. 1989 "Bidirectional use of knowledge in the multi-modal NL access system XTRA", Proceedings of the 11th International Joint Conference on Artificial Intelligence, 1492-1497.

Appelt, D. E. 1987 "Bidirectional Grammars and the Design of Natural Language Generation Systems," In Y. Wilks (ed.) Theoretical Issues in Natural Processing-3, New Mexico State University, Las Cruces, New Mexico, 185-191.

Busemann, S. and Hauenschild, C. 1988 "A Constructive View of GPSG or How to Make It Work," Proceedings of the 12 in International Conference on Computational Linguistics, 77-82.

Dymetman, M. and Isabelle, P. 1988 "Reversible Logic Grammars for Machine Translation," Proceedings of the 2nd International Conference on Theoretical and Methodological Issues in Machine Translation of Natural Language.

Dymetman, M.; Isabelle P. and Perrault, F. 1990

"A Symmetrical Approach to Parsing and Generation,"

Proceedings of the 13th International Conference on Computational Linguistics, 90-96.

Finkler, W. and Neumann, G. 1989 "POPELHOW: A Distributed Parallel Model for Incremental Natural Language Production with Feedback," Proceedings of the Eleventh International Joint Conference on Artificial Intelligence, 1518-1523.

Hocppner, W.; Christaller, T.; Marburger, H.; Morik, K.; Nebel, B.; O'Leary, M. and Wahlster, W. 1983 "Beyond Domain-Independence: Experience with the Development of a German Language Access System To Higly Diverse Background Systems," Proceedings of the 8th International Joint Conference on Artificial Intelligence, 643-646.

Horacek, H. and Pyka, C. 1988 "Anwendbarkeit von Unifikationsgrammatiken für effizientes Generieren," In H. Trost (ed.) 4.Osterreichische Artificial-Intelligence-Tagung, Springer, Berlin, 171177.

Jackendoff, R. 1987 "Consciousness and the Computational Mind," Cambridge Massachussetts: MIT Press.

Jacobs, P. S. 1988 "Achieving Bidirectionality," Proceedings of the 12th International Conference on Computational Linguistics, 267-274.

Koskenniemi, K. 1984 "A General Computational Model for Word-Form Recognition and Production," Proceedings of the 10th International Conference on Computational Linguistics, 178-181.

Lancel, J.M.; Otani, M.; Simonin, N. and Danlos, L. 1988 "SAGE: a Sentence Parsing and Generation System," Proceedings of the 12th International Conference on Computational Linguistics, 359-364.
Mann, W. C. 1987 "What is Special About Natural Language Generation Research?," In Y. Wilks (ed.) Theoretical Issues in Natural Processing3, New Mexico State University, Las Cruces, New Mexico, 206-211.

McDonald D. D. 1987 "No Better, but no Worse, than People," In Y. Wilks (ed.) Theoretical Issues in Natural Processing-3, New Mexico State University, Las Cruces, New Mexico, 200-205.

McDonald, D. D.; Meteer, M. W. and Pustejovsky, J. D. 1987 "Factors Contributing to Efficiency in Natural Language Generation," In G. Kempen (ed.) Natural Language Generation: New Results in Artificial Intelligence, Psychology and Linguistics, Dordrecht: Martinus Nijhoff, 159-182.

Neumann, G. and Finkler, W. 1990 "A Head. Driven Approach to Incremental and Parallel Generation of Syntactic Structures," Proceedings of the 13th International Conference on Computational Linguistics, 288-293.

van Noord, G. 1990 "Reversible Unification Based Machine Translation," Proceedings of the 13th International Conference on Compulational Linguistics, 299-304.

Pereira, F. C. N. and Warren, D. H. D. 1983

"Parsing as Deduction, "Proceedings of the 21th Annual Meeting of the Association for Computational Linguistics, 137-144.

Proudian, D. and Pollard, C. 1985 "Parsing Head. Driven Phrase Structure Grammar, "Proceedings of the 23rd Annual Meeting of the Association for Computational Linguistics, 167-171.

Pollard, C. and Sag, I. 1987 "Information-based syntax and semantics," CLSI Lecture Notes 13, Center for the Study of Language and Information, Standford, CA.

Shieber, S. M. 1988 "A Uniform Architecture for Parsing and Generation," Proceedings of the $12 \mathrm{th}$ International Conference on Computational Linguistics, 614-619.

Shieber, S.; M.; van Noord, G.; Moore, R. M. and Pereira, F. C. P. 1989 "A Semantic Head-Driven Generation Algorithm for Unification-Based Formalisms," Proceedings of the 27th Annual Meeting of the Association for Computational Linguistics, 7-17.

Uszkoreit, H. 1991 "Strategies for Adding Control Information to Declarative Grammars," Technical Reporl, Institute for Computational Linguistics, University of Saarbrücken, FRG.

Zajac, R. and Emele, M. 1990 "Typed Unification Grammars," Proceedings of the 13th International Conference on Computational Linguistics, 293-298. 